\title{
A Fuzzy Decision Table Approach for Business Site Selection
}

\author{
Geert WETS $^{\mathrm{a}}$, Frank WITLOX $^{\mathrm{b}}$, Harry TIMMERMANS ${ }^{\mathrm{c}}$ \& Jan VANTHIENEN ${ }^{\mathrm{a}}$ \\ ${ }^{a}$ Katholieke Universiteit Leuven, Department of Applied Economic Sciences, Naamsestraat 69, \\ B-3000 Leuven - Belgium, E-mail: \{geert.wets, jan.vanthienen\}@econ.kuleuven.ac.be \\ ${ }^{b}$ University of Antwerp (UFSIA), Faculty of Law, Department of Economic and Social Sciences, \\ Rodestraat 14 (R 204), B-2000 Antwerp (Belgium), E-mail: frw.witlox.f@alpha.ufsia.ac.be \\ ${ }^{\mathrm{c}}$ Eindhoven University of Technology, Faculty of Architecture, Building and Planning, Department of \\ Architecture and Urban Planning, P.O. Box 513, Mail station 20, NL-5600 MB Eindhoven \\ (The Netherlands), E-mail: h.j.p.timmermans@bwk.tue.nl
}

\begin{abstract}
In this paper it is explained how the techniques of fuzzy decision tables can used for business site selection. It is outlined how this technique fits in the spectrum of techniques which can be adopted for business site selection. Furthermore, it is described how decision tables can be modelled based on functional equivalence. The decision tables are modelled with a tool called Prologa. This tool supports the interactive construction, optimization and consultation of knowledge structuring formalisms such as decision tables, decision rules and decision trees.
\end{abstract}

\section{Introduction}

The problem of locational choice modelling is a well explored subject not only among geographers, planners and economists but it also recently gained the interest from fuzzy researchers. This has lead to the promising development that a number of standard quantitative and qualitative locational modelling techniques are being extended with notions from fuzzy set theory.

The principle idea is that when firms are evaluating potential location sites they use imprecise and vague notions when describing their locational decision-making process. Because fuzziness is abound in human choice making, applying fuzzy set theory allows one to deal with this vagueness in a more precise way. Crisp decision tables only appear to produce an accurate or non-fuzzy decision output because the imprecision inherent in decision-making is not noticed. Therefore, in this contribution, we advance the technique of fuzzy decision tables as an interesting extension to the qualitative research methods of crisp decision tables.

The paper is organized as follows. In section 2 the crisp decision table representation is briefly described. In section 3 different approaches to locational choice models are given. The next section concentrates on decision tables and functional classification. Section 5 of this paper defines the extension of the crisp decision table to include fuzziness. In section 6 it is demonstrated how decision tables can be used in locational choice. Next, some issues about the consultation of fuzzy decision tables are specified. Finally, our major findings are summarized, and some suggestions for future research paths are given.

\section{Crisp decision tables}

A decision table is a tabular representation used to describe and analyze procedural decision situations, where the state of a number of conditions determines the execution of a set of actions. Not just any representation, however, but one in which all distinct situations are shown as columns in a table, such that every possible case is included in one and only one column.

The tabular representation of the decision situation is characterized by the separation between conditions and actions, on one hand, and between subjects and conditional expressions (states), on the other. Every table column (decision column) indicates which actions should (or should not) be executed for a specific combination of condition states. In order to make a meaningful use of decision tables possible, the decision table has to be defined clearly and must meet the important requirements of consistency and completeness.

A decision table contains four parts: condition subjects, condition states, action subjects and action values. These four parts can be defined more formally: 
Definition 1. Let $\mathrm{CS}_{\mathrm{i}}$ be a condition subject with domain $\mathrm{CD}_{\mathrm{i}}(\mathrm{i}=1, \ldots$, cnum $), \mathrm{CT}_{\mathrm{i}}$ be a set of condition states $S_{i k}\left(k=1, \ldots, n_{i}, i=1, \ldots\right.$, cnum $)$ with $S_{i k}$ being a logic expression, $A S_{j}(j=1$..anum) be an action subject; and $A V_{j}=\{$ true $(x)$, false (-), nil (.) $\}$ be an action value set $(\mathrm{j}=1, \ldots$, anum), then a decision table is a function from $\mathrm{CT}_{1} \times \mathrm{CT}_{2} \times \ldots \times \mathrm{CT}_{\text {cnum }}$ to $\mathrm{AV}_{1} \times \mathrm{AV}_{2} \times \ldots \mathrm{x}$ $A V_{\text {anum }}$ such that each possible condition combination is mapped into one (completeness or exhaustivity) and only one (exclusivity) action configuration.

Notably, the elements of $\mathrm{CD}_{\mathrm{i}}$ involved in a condition state $S_{i k}$ determine a subset of $C D_{i}$, such that the set of all these subsets constitutes a partition of $C D_{i}$.

The use of decision tables has several advantages. Firstly, they can be easily verified and validated (V\&V) [18]. Subsequently, they provide a structuring formalism which can be used efficiently in the knowledge acquisition phase [16]. Finally, decision tables can be executed very efficiently [7]. A major problem of the use of decision tables, however, is the complexity of the manual building process. Therefore, Prologa (PROcedural LOGic Analyzer) has been developed. This is an interactive design tool for computer-supported construction and manipulation of decision tables [17]. When building decision tables, the designer essentially provides the system with the following information: a list of conditions with their states, a list of actions and a list of rules. This will enable the system to construct the corresponding decision tables. During this process, a V\&V check will take place [20]. After this process the decision tables can be consulted. This can be accomplished either visually or by transforming the decision tables to a format that can be executed in an expert system shell or program [16].

\section{Business site location models}

There are many different methods available to analyse the problem of business site selection. Often, in the literature on location modelling [15], [22] a distinction is made between quantitative approaches and qualitative techniques.

On the one hand, the quantitative location choice models, such as the spatial interaction models, revealed preference models, discrete choice models, and more recent, the so-called decompositional multiattribute preference and choice models (also known as conjoint analysis or stated choice models), all focus on trying to explain and predict a firm's choice in respect to different business location sites. The aggregate spatial interaction models (i.e., gravity models) were used to delimit a certain market area whereby it was assumed that two towns attract firms from an intermediate town in direct proportion to the populations of the two cities and in inverse proportion to the squares of the distances from these two town to the intermediate town. Because a number of problems are associated with these gravity models, the focus shifted from the delimitation of market areas to probabilities of interaction and disaggregate choice modelling. A new modelling technique came to the fore, known as revealed preference models and discrete choice models which originated from behavioural geography and psychology.

It is typical of this approach to make use of probabilistic choice models in an attempt to characterize different patterns of choice behaviour of firms. The location decision is usually modelled as a selection procedure in terms of a number of site alternatives that are defined by means of a number of attributes that mathematically combined result in a certain utility. The alternative with the highest utility is of course selected. The way in which the attributes are combined to form the overall utility of the alternative makes it possible to distinguish between fundamental measurement and conjoint measurement. In both cases a multinomial logit model could be used to estimate the site alternative's utilities.

On the other hand, qualitative models are typically concerned with trying to uncover which factors influence the decision-making process and with reconstructing the process of how a decision or choice option was finally made. Therefore, qualitative models such as decision plan nets, decision trees, and decision tables function more as decision support systems that will assist firms when they are making a business site selection. It is characteristic of qualitative models that they are less based on the use of statistical and mathematical procedures to arrive at a certain solution.

In recent years, however, it can be noted that fuzzy set theory is being introduced in both the quantitative and, to a some lesser extent, qualitative approaches to business site location modelling. Both approaches are making use of the fuzzy set extensions in order to deal with vagueness and imprecision that are abound in human decisionmaking. In quantitative methods such techniques are being suggested as fuzzy linear programming, fuzzy parametric analysis, and fuzzy simulation (e.g. [1], [2], [3], [6]). In the qualitative approach applications are to our knowledge rather limited [10], [13]. Therefore, in this contribution it is our aim to advance the qualitative technique of the decision table which incorporates elements of fuzzy set theory. The result is a so-called fuzzy decision table (FDT).

\section{Decision tables and functional classification}

In functional modelling, choice behaviour is viewed as being highly goal-oriented whereby these goals can be achieved through a variety of functional equivalent strategies. Spatial choice is not analyzed on the basis of an actor's preferences, but on the basis of the relationship between an object and its context. Such an 'end-means' 
relationship automatically implies goal-rationality. Or, to put it in other words: "What is important is not to find the object [i.e., the choice alternative] with the highest preference value, but the object or set of objects that can satisfy a pre-defined goal or objective" [12].

An important factor in relational modelling is that a distinction is made between an object-type and an object on the basis of the intension and extension of concepts. The intension of a concept refers to a set of necessary and sufficient conditions that should be satisfied by an object in order that the object may be classified under the particular concept. Therefore, the intension of a concept refers to the object-type, as it sets the scene for evaluating whether an object is or is not to be considered to belong to the class covered by the concept. The extension of the concept points to a set of objects that comply with the object-type. A simple example may clarify this distinction somewhat further. Take, for instance, a concept like 'bachelor'. The intension of this concept is determined by three necessary conditions that put together are sufficient: 'male', 'adult', and 'not married'. Only those persons that comply with all three conditions, are considered as extensions of the concept 'bachelor'. Persons that do not match with all three conditions cannot accurately (and also literally) be classified as extensions of the concept 'bachelor'. For instance, a person that lacks the 'unmarried' condition might be termed a 'man', and if the 'married' condition is present, a 'husband'. Clearly, the importance of a distinction between object-type and object has everything to do with a matching or classification problem, in the sense that constraints defined by the object-type need to be matched by a set of attributes defining an object in order that the object can be classified as an object-type.

In the literature [14] several theories have been put forward that can be used to interpret and solve this matching or classification problem. In the classical theory, classification of objects as object-types is established on a simple crisp, 'yes' or 'no' (all or nothing) basis, in the sense that an object either complies or does not comply with the object-type. Characteristic for such a theory is the assumption that the set of necessary and sufficient conditions defining the object-type is totally univocal, which automatically implies a totally unambiguous matching. If this were true, matching or classification would be very straightforward. However, intuitively, it stands to reason that classification is far more complex than that the classical theory perceives it to be. As a consequence, a number of alternative theories have been developed that assumed that classification problems result from a sort of "fuzziness" in the delineation of the set of constraints that define an object-type. One of those theories is called the functional classification theory which aims at explicitly expressing the methodological viewpoint of relational realism.
The central notion of functional classification is that on a theoretical level an object-type cannot be univocally defined through a set of constraints due to some sort of fuzziness. It is assumed that fuzziness has a systematic character. Therefore, it cannot be avoided either by the elimination of random disturbances through some mathematical technique or by comparing the objects to a prototypical object-type. Thus, the problem of fuzziness should be solved in another way. The solution is found by consequent allowing for the systematic identification and reconstruction of several object-types instead of one single object-type. In this respect, matching or classification is based on the so-called functionality of the objects. This means that objects, although they have different sets of attributes, are assigned to distinct classes if they can fulfil specific functions associated with these classes. In the functional classification theory this characteristic is referred to as functional equivalence, or "the phenomenon that objects, possibly differing in many respects, are equivalent in achieving a nominally specified function in a certain context" [11].

Applied to the locational choice problem of businesses, the object refers to a 'production environment' and the object-type to a 'potential location site'. The production environment is any geographical unit, be it a region, city, district, enterprise zone, etc., that is eligible or suitable to serve as a potential location site for a particular company. The production environment is characterized by a variety of location factors (attraction and repulsion forces) that combined form a kind of "locational profile" (i.e., the supply-side). This profile needs to be matched with the specific locational requirements put forward by the individual firm (i.e., the demand-side) that should be present in order to achieve the goal of finding the most suitable location site.

It follows from the above that a production environment can only function as a potential location site for a firm, if the characteristics of the production environment relationally match with the characteristics of the production requirements put forward by the firm. Only when both properties match successfully, the production environment is able to fulfil the function of potential location site for the firm, and can be classified accordingly. In all likelihood, more than one production environment will be classified as suitable location site. Therefore, the result of the matching process is a set of functional equivalent production environments from which a selection can be made. Thus matching is defined as a two-way process based on functional equivalence, and a knowledge representation formalism, like decision tables, complies with that definition. 


\section{Fuzzy decision tables}

It is evident that crisp decision tables produce crisp decision outputs. This property was deemed to be one of the major strengths of applying the crisp decision table formalism. However, it was also noted that the greatest weakness of crisp decision making is the fact that the crisp decision table is unable to take into account imprecisions and uncertainties that are abound in human decision making. In a way, the price to pay for the pursuit of accuracy is the lack of being able to allow for flexibility. In many problems crisp decision tables prove to be too stringent. Therefore, based on recent progress in crisp decision table formulation and standardization [16], [18], [19], [20] we have enhanced the decision table formalism with some fuzzy concepts [4], [5]. In the remainder of this section the basic notions about fuzzy decision tables are outlined.

A crisp decision table may be extended to include fuzziness in the condition part and/or in the action part, which then gives rise to the notion of a fuzzy decision table. Fuzziness in the condition part can be expressed by fuzzy conditions (in form of simple predicates) such as "Distance is long" while fuzziness in the action part can be expressed by linguistic terms such as "Value of land is high". In a fuzzy decision table, these linguistic terms and fuzzy sets appear with condition states $\left(S_{i k}\right)$ and/or with action subjects $\left(\mathrm{AS}_{\mathfrak{j}}\right)$. More formally, a fuzzy decision table is defined as follows:

Definition 2. Let $\mathrm{CS}_{\mathrm{i}}$ be a condition subject with domain $\mathrm{CD}_{\mathrm{i}}(\mathrm{i}=1, \ldots$, cnum $), C \mathrm{CT}_{\mathrm{i}}$ be a set of condition states $S_{i k}\left(k=1, \ldots, n_{i}, i=1, \ldots\right.$, cnum $)$ with $S_{i k}$ being a fuzzy logic expression, $A S_{j}$ be an action subject incorporated with linguistic terms and fuzzy sets, and $A V_{j}$ $=\{$ true $(x)$, false $(-)$, nil $()$.$\} be an action value set (j=1$, $\ldots$, anum), then a fuzzy decision table is a function from $\mathrm{CT}_{1} \times \mathrm{CT}_{2} \times \ldots \times \mathrm{CT}_{\text {cnum }}$ to $\mathrm{AV}_{1} \times \mathrm{AV}_{2} \times \ldots \times \mathrm{AV}_{\text {anum }}$ such that each possible condition combination is mapped into one action configuration.

An example of a fuzzy decision table, in the field of location theory, is shown in Figure 1. This fuzzy decision table was built with the decision table workbench Prologa.

\begin{tabular}{|l|c|c|c|c|}
\hline 1. Distance to railway station & short & \multicolumn{3}{|c|}{ long } \\
\hline 2. Distance to harbor & - & short & about average & long \\
\hline 1. Accessibility is good & $x$ & $x$ & & \\
\hline 2. Accessibilily is medium & & & $x$ & \\
\hline 3. Accessibility is bad & & & & $x$ \\
\hline & 1 & 2 & 3 & 4 \\
\hline
\end{tabular}

Figure 1: A fuzzy decision table
The construction of fuzzy decision tables can proceed mainly according to the steps of the crisp case, however, some extensions are needed (see also below). For example, extra steps are necessary to specify fuzzy sets involved in condition or actions, some provisions are needed to handle fuzzy decision rules, etc.

As far as the properties of decision tables (completeness, exclusivity, correctness) are concerned, it can be seen that definition 2 guarantees the completeness because any possible condition combination will lead to a decision in terms of action configurations.

The property of exclusivity, however, needs to be relaxed since it is no longer the case that there exists one and only one perfect match. In the fuzzy case the degree of matching between a column in a decision table and a given condition configuration is a value in $[0,1]$. This, however, should not be a problem since the nature of fuzziness allows for some overlap between states. This does not mean that the $\mathrm{V} \& \mathrm{~V}$ process becomes obsolete in fuzzy systems [8], [9].

The notion of correctness can be determined in a similar way to that of the crisp case. That is, it can be checked by the knowledge engineer whether the fuzzy decision table reflects the ideas of the expert.

\section{Fuzzy decision tables and locational choice}

To illustrate how fuzzy decision tables can represent a two-way matching process based on functional equivalence in the field of locational choice [21], we refer to Figure 2. In this figure, three fuzzy decision tables (i.e., one output table and two input tables) are combined in order to evaluate the labour market conditions (L.M.C.) of a potential location site for a particular business. The output table (i.e., the top table) represents the quality of the two-way relational match. This table is constructed on the basis of the outcome of the two input tables. Note that different categories of matching are possible, and that it is only when the required L.M.C. equals the available L.M.C. that the matching process is termed "ideal". Consequently, over or under matching results from a discrepancy in the required L.M.C. demanded by a particular company and the available L.M.C. present at a particular location site.

Note, however, that a decision cannot be taken by merely checking with each column of the table to match perfectly a given condition configuration. Instead, the degree of matching between the given condition combination and each column should be evaluated [4]. As a result, more than one action configuration may be chosen, each with a degree in $[0,1]$. 


\begin{tabular}{|c|c|c|c|c|c|c|c|c|c|}
\hline 1. ^required L.M.C & \multicolumn{3}{|c|}{ low } & \multicolumn{3}{|c|}{ medium } & \multicolumn{3}{|c|}{ high } \\
\hline 2. Aavailable L.M.C & low & medium & high & low & medium & high & low & medium & high \\
\hline 1. ideal malching & $x$ & & & & $\mathrm{~s}$ & & . & . & $x$ \\
\hline 2. over matching & & $\mathrm{x}$ & & & & $\mathrm{x}$ & & & \\
\hline 3. strong over matching & & & $\mathrm{x}$ & & & & & & \\
\hline 4. under matching & & & & $\mathrm{x}$ & & & . & $x$ & \\
\hline 5. strong under matching & & & . & & & & $x$ & . & \\
\hline
\end{tabular}

\begin{tabular}{|c|c|c|c|c|c|c|}
\hline 1. skilled labour & & & & $Y$ & & $\mathbf{N}$ \\
\hline 2. unionization rate & & $<30 \%$ & & $y=30 \%$ & & $\cdot$ \\
\hline 3. produclivily rate & low & medium & high & low of medium & high & - \\
\hline 1. required L.M.C is low & $\mathrm{x}$ & . & . & . & . & $x$ \\
\hline 2. required L.M.C is medium & & $x$ & & $x$ & . & . \\
\hline 3. required L.M.C is high & . & . & $x$ & . & $x$ & \\
\hline
\end{tabular}

\begin{tabular}{|c|c|c|c|c|c|}
\hline 1. educational training & low & \multicolumn{4}{|c|}{ medium or high } \\
\hline 2. productivity rate & & \multicolumn{2}{|c|}{ low } & \multicolumn{2}{|c|}{ high } \\
\hline 3. wage rate & - & $<\$ 25000$ & $\gamma=\$ 25000$ & $<\$ 25000$ & \rangle$=\$ 25000$ \\
\hline 1. available L.M.C is low & $x$ & 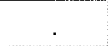 & $x$ & . & \\
\hline 2. available L.M.C. is medium & . & $x$ & . & . & $x$ \\
\hline 3. arailable L.M.C. is high & . & & . & $x$ & \\
\hline
\end{tabular}

Figure 2: Example of two-way matching of labour market conditions

Obviously, in a real business location problem other factors need to be evaluated as well such as transportation conditions, agglomeration economies, availability of public utilities, etc. For these locational factors, however, an identical matching process can be applied.

\section{Fuzzy consultation}

Two major categories for consulting fuzzy decison tables can be distinguished (1. calculating the degree of matching between a column in the decision table and a given condition combination; 2 . transforming the columns in a decision table to IF-THEN rules which can then be consulted in an expert sytem shell). In [4] it was explained technically how these two methods can be applied to consult fuzzy decision tables. More concretely in the domain of location choice modelling the following remarks can be made.

When comparing the crisp and fuzzy decision output, it should be clear that the latter allows for a more subtle decision differentiation than the former. This is because crisp decision tables are unable to make comparisons between location sites that point to an identical crisp action state. The table evaluates all these locations as functionally equivalent, but makes no further distinction in terms of a degree of matching or ranking order. Recall that it was exactly this lack of flexibility typical of working with crisp decision tables that gave cause to the introduction of fuzziness in the decision making process.

In contrast to the crisp decision table output, the fuzzy decision table produces for each potential location site a membership value with the fuzzy set of suitable locations. In other words, the matching process will by no means always be evaluated as "ideal". As a result, it is possible to compare and rank location sites in terms of their overall calculated membership values whereby it is obvious that the higher the membership value, the better the degree of matching, and vice versa. Note also that only those locations that have similar membership values are deemed totally functional equivalent.

In conclusion, it may appear that the construction and consultation of a fuzzy decision table is a rather timeconsuming process because all decision rules of the crisp decision table need to be specified in terms of membership functions and values. This is however wrong. After all, whenever a zero membership value is calculated for a particular condition state, this implies that all subsequent condition states need not to be determined as they will have no influence on the fuzzy decision output.

For the moment, one major drawback of fuzzy consultation, is that the semantics of it are poorly understood. The current state of the art of fuzzy set theory provides little help on this issue. Especially, when reasoning in a knowledge based system it is very difficult to determine which implication function should be chosen in order to produce the desired outcome.

\section{Conclusions and future research}

In this paper fuzzy decision tables were defined and constructed. Moreover, it was explained how they can be applied in the field of locational choice modelling. Currently, we are using fuzzy decision tables to model a locational choice problem of a petro-chemical industrial plant. One of the main topics of interest in this project is to evaluate the influence of the different implication operators on the consultation process of fuzzy decision tables. Further topics for future research include the interpretation of fuzzy membership functions in the specific context of location choice modelling and V\&V of the fuzzy decision tables.

\section{References}

[1] Banai-Kashani A.R., Dealing with uncertainty and fuzziness in development planning: a simulation of high-technology industrial location decision making by the analytic hierarchy process, Environment and Planning A 22 (1990) 1183-1203. 
[2] Bhattacharya U., Rao J.R. and Tiwari R.N., Fuzzy multicriteria facility location problem, Fuzzy Sets and Systems 51 (1992) 277-287.

[3] Bhattacharya U., Rao J.R. and Tiwari R.N., Bi-criteria multi facility location problem in fuzzy environment, Fuzzy Sets and Systems 56 (1993) 145-153.

[4] Chen G., Vanthienen J. and Wets G., Fuzzy decision tables: extending the classical formalism to enhance intelligent decision making. International Joint Conference of the 4th IEEE International Conference on Fuzzy Systems and the 2nd International Fuzzy Engineering Symposium (FUZZ-IEEE/IFES'95), March 20-24, Yokomama, Japan, (1995) 599-606.

[5] Chen G., Vanthienen J. and Wets G., Representing fuzzy decision tables in a fuzzy relational database environment, Third European Congress on Intelligent Techniques and Soft Computing (EUFIT'95), August 28-31, Aachen, 1995, 697-700.

[6] Chung K.-H. and Tcha D.W., A fuzzy set-theoretic method for public facility location, European Journal of Operational Research 58 (1992) 90-98.

[7] Colomb R. and Chung C., Strategies for building propositional expert systems, International Journal of Intelligent Systems 10 (1995) 295-328.

[8] Gottwald S. and Petri U., An algorithmic approach toward consistency checking for systems of fuzzy control rules, Third European Congress on Intelligent Techniques and Soft Computing (EUFIT'95), August 28-31, Aachen, 1995, 682-687.

[9] Kinkielélé D. and Ayel M., On discovering potential inconsistencies in validating fuzzy knowledge bases, Fifth International Conference on Information Processing and Management of Uncertainty in Knowledge Based Systems (IPMU'94), July, Paris, 533-539.

[10] Liang G.-S. and Wang M.-J.J., A fuzzy multi-criteria decision-making method for facility site selection, International Journal of Production Research 29 (1991) 2313-2330.

[11] Lucardie G.L. Functional object-types as a foundation of complex knowledge-based systems, Rijswijk, TNO Bouw, Ph.D. thesis, 1994.

[12] Reitsma R.F., Functional classification of space; aspects of site suitability assessment in a decision support environment, Laxenburg, International Institute for Applied Systems Analysis, 1990.

[13] Skikos G.D., Applying fuzzy set theory to evaluate and select potential sites for wind turbine installations, Wind Engineering 18 (1994) 283-295.

[14] Smithson M., Fuzzy set analysis for behavioral and social sciences, New York, Springer Verlag, 1987.

[15] Timmermans H., Retail environments and spatial shopping behavior, In: Gärling, T. and Golledge, R.G. Eds Behavior and Environment; Psychological and Geographical Approaches. Amsterdam, Elsevier Science Publishers, 1993, 342-377.

[16] Vanthienen J. and Wets G., From decision tables to expert system shells, Data \& Knowledge Engineering 13 (1994) 265-282.
[17] Vanthienen J. and Dries E., Illustration of a decision table tool for specifying and implementing knowledge based systems, International Journal on Artificial Intelligence Tools 3 (1994) 267-288.

[18] Vanthienen J., Aerts A., Mues C. and Wets G., A modelling approach to KBS verification, EUROVAV95, Chambéry, France, 1995, 155-171.

[19] Vanthienen J. and Wets G., Integration of a decision table system with a relational database environment, Information Systems 20 (1995) 595-616.

[20] Vanthienen J. and Dries E., Decision tables: refining the concept and a proposed standard, to appear in: Communications of the ACM.

[21] Wets G., Witlox F., Timmermans H. and Vanthienen J., Locational choice modelling using fuzzy decision tables, NAFIPS'96, Berkeley, (1996) 80-84.

[22] Witlox F., Qualitative housing choice Modelling: decision plann nets versus decision tables, Netherlands Journal of Housing and the Built Environment 10 (1995) 209-237. 